\title{
Improving Energy Efficiency of Grouping Abstraction in Cyber Physical System
}

\author{
Pooja Sobhrajan \\ Computer Engineering Department \\ DYPIET Pimpri, Pune
}

\author{
Swati Y Nikam \\ Computer Engineering Department \\ DYPIET Pimpri, Pune
}

\begin{abstract}
Cyber Physical System (CPS) integrates physical and cyber components for high performance, self-maintenance, selforganization, self-assembly, while the process must be dependable, safe, secure, and efficient in real-time. It supports heterogeneous devices, such as motes, PDAs, laptops, and actuators according to the applications' requirements. This paper describes an improved grouping abstraction for Cyberphysical systems. This abstraction allows different applications to simultaneously use the same sensors and actuators. It facilitates feedback control mechanisms by dynamic membership update and requirements reconfiguration based on feedback from the current members. It is implemented in Java, which ensures easy and conciseness of programming. It facilitates in-network aggregation and local processing which improves the lifetime of the network. This is shown in result to reduce energy consumption for enhancing the lifetime of the network. It would be validated on the application of smart campus, which takes care of three functions like classroom occupancy, attendance monitoring aand area access controlling and monitoring.
\end{abstract}

\section{General Term}

Cyber Physical System

\section{Keywords}

Sensor network, Cyber Physical System, Actuator, communications, computation, programming, software.

\section{INTRODUCTION}

Using the Internet we can interact with people and get useful information from the wide world in a very short time. Thus, the Internet has transformed the way we do our research, studies, we manage our business and services and even our fun way. However, there is a gap between the cyber world in which information is transmitted, modified and the physical world in which we live. Also, the last two decades have brought a digital revolution that has transformed the industry. This change is not a choice, but it is determined by fundamental, economic and technological long term trends, that have created an environment which allows and requires a wide and varied range of new capabilities [14].

The technological advances from the recent decades has led to some early examples of a new system generation (e.g. quality, safety and efficiency critical infrastructure; integrated, self optimizing transportation systems and vehicles; airplanes and automobiles that are environmentally friendly and energy efficient; advanced health care via increased automation, integrating smart devices, and providing safe access to electronic medical records; new biotechnologies). These early examples demonstrate the need for a new generation of systems - Cyber-Physical Systems (CPSs) - to represent more than networking and information technology, information and knowledge being integrated into physical objects. By integrating perception, communication, learning, behaviour generation, reasoning into such systems a new generation of intelligent and autonomous systems is to be developed. We have surveyed the research challenges of cyber physical system in [15]. There are different types of programming abstractions in cyber physical system, but we are focusing on the concept of grouping abstraction in cyber physical system.

This paper is organized as follows. Section 2 describes grouping abstraction in detail. Section 3 explains the problem domain. Section 4 describes proposed idea. Section 5 describes a mathematical model of the proposed system. Section 6 describes design and section 7 its implementation details. Section 8 and 9 presents its evaluation results and result analysis. The conclusion is given in Section 10.

\section{GROUPING ABSTRACTION}

Cyber-physical systems (CPSs) become widespread include heterogeneous sensing and actuation devices, support intra and internetwork mobility, permit multiple applications to execute simultaneously and be accessible and controllable via the Internet. Ubiquitously deployed wireless sensor networks (WSNs) enhanced with actuators will create a new CPS infrastructure and along with body networks and sensor-based cell phones will create a situation with many interacting systems of systems. For this vision to become common place abstractions are required that support ease of programming, grouping sensors, and actuators of different kinds from different networks and administrative domains, and dynamically managing these groups in the presence of mobility and feedback control.

A group based abstraction with important capabilities for across system programming, mobility, automatic dynamic updates, fine grained access right control and conflict resolution mechanism and support for actuators. It allows grouping devices based on the services they offer and their location information. It is a high level programming language that allows operations to be performed on the chosen subset through code migration.

\section{PROBLEM CHARACTERIZATION}

Although the group based programming abstraction for cyber physical system is a powerful and flexible programming abstraction, but there are some limitations, mainly due to its centralized architecture which are as follows:-

\subsection{In-network aggregation and local processing}

It does not have the capability of in-network aggregation and processing. This results in more energy consumption than the distributed programming abstraction in some specific scenarios.

\subsection{Response time}

All the configuration messages from the applications must be first uploaded to the negotiator. After being processed, these 
messages go from the negotiator to the network nodes via the gateway. The updates from the network nodes also have to go to the negotiators first, from where they eventually reach the applications. Therefore, the response time is not as good as the other programming abstractions.

\subsection{Dependency}

Another limitation is that they create a strong dependency between the negotiators and the resource constrained sensors: the sensors must be able to communicate with their negotiator in order to configure themselves properly and store the data they generate [1].

\section{PROPOSED IDEA}

Current research areas in Cyber Physical Systems are energy control, secure control, transmission and management, control technique and grouping abstraction. The objective is to make improvement in grouping abstraction for Cyber physical system. Therefore, the design, implementation and evaluation of an agent based grouping abstraction are as follows:

\subsection{Reduce Energy Consumption}

To support in-network aggregation and processing for increasing the lifetime of the network.

\subsection{Improving Response Time}

To explore feasible solutions of an efficient message management system so as to improve the response time.

\subsection{Independence}

It would be independent on any other module.

\section{Mathematical Model}

The energy used by a node consists of the energy consumed by computing, receiving and transmitting. The switching of state can also cause significant energy consumption. In the calculation of these operational energy costs in a sensor node, consider the MAC protocol, as it has a significant impact on energy consumption. In calculating energy consumption, use the maximum values of parameters for the worst case analysis.

\subsection{Sensing Energy}

Due to the wide diversity of sensors, the power consumption of sensors varies greatly. In general, a sensor, i, will have the following sensing energy consumption.

$$
\mathrm{Esm}=\mathrm{Vdc} * \mathrm{Ii} * \mathrm{Ti}
$$

where $\mathrm{Ti}$ is the time required for obtaining a single sample from sensor $\mathrm{i}$ and Ii is the current draw of sensor $\mathrm{i}$. Ti depends on the start-up (Ts), response (Tr) and measurement (Tm) times of the sensors. As Tm is small in comparison to Ts and $\operatorname{Tr}$ for most sensors, we consider only $\mathrm{Ts}$ and $\mathrm{Tr}$ in calculating Ti.

\subsection{Computational Energy}

The computational energy cost (Ecomp) of sensor motes is a key constituent of the overall operational energy costs. Ecomp includes the MCU's active mode and other modes' (e.g., standby/idle/sleep) energy consumption. In cases of complex mathematical operations Ecomp can be expressed as:

Ecomp $=\mathrm{Vdc} *$ Imcu-active $*$ Tmcu-active $+\mathrm{Vdc} *$ Imcusleep * Tmcu-sleep

where Imcu-active and Imcu-sleep are the MCU active and sleep mode current, respectively. Tmcu-active and Tmcusleep are the MCU active and sleep modes durations, respectively.

\subsection{Communication Energy}

The constituents of Ecomm are listening, transmission, reception, sleeping and switching energy. The transmission energy, Etx, component of Ecomm refers to the energy consumed during the transmission of packets. Etx can be expressed as:

$$
\mathrm{Etx}=\mathrm{Vdc} * \mathrm{Itx} * \mathrm{~Pb} * \mathrm{~Tb}
$$

where Itx is the current consumption in the transmission mode of the radio. $\mathrm{Pb}$ is the bit length of the packet to be transmitted and $\mathrm{Tb}$ is the transmission time of a single bit.

The reception energy, Erx, component of Ecomm refers to the energy consumed when receiving packets. Erx can be expressed as:

$$
\operatorname{Erx}=\mathrm{Vdc} * \operatorname{Irx} * \mathrm{Pbr}^{*} \mathrm{~Tb}
$$

where Irx is the current consumption in reception mode and $\mathrm{Pbr}$ is the bit length of the packet to be received.

The listening energy, Elisten, is the radio energy consumption when the radio is active, but not receiving or sending packets. This listening is to check for messages low power listening. Elisten can be expressed as:

$$
\text { Elisten }=\mathrm{Vdc} * \text { Ilisten } * \text { Tlisten }
$$

where Ilisten is the current draw in listen mode and Tlisten is the time in each sampling. The following equation determines the energy consumed for the switching state:

$$
\text { Esw }=(\mid(\text { Istj }- \text { Isti }) \mid * \text { Tstij } * \text { Vdc }) / 2
$$

where Istj is the current draw of the radio in the state switched to, and Isti is the current draw of the radio in the current state and Tstij is the time required for the radio to go from state $i$ to j. If a radio switches from sleep mode to transmit or receive mode, it uses wake-up-time as Tstij otherwise, it is the switching-time.

\subsection{Response Time}

The response time is in direct proportion to the wait time. The relationship among response, wait and processing time is:

Response time $=$ wait time + processing time

\section{DESIGN}

To overcome the limitations characterized in the section 3, we present the proposed architecture and case study of Smart Campus. 


\subsection{System Architecture}

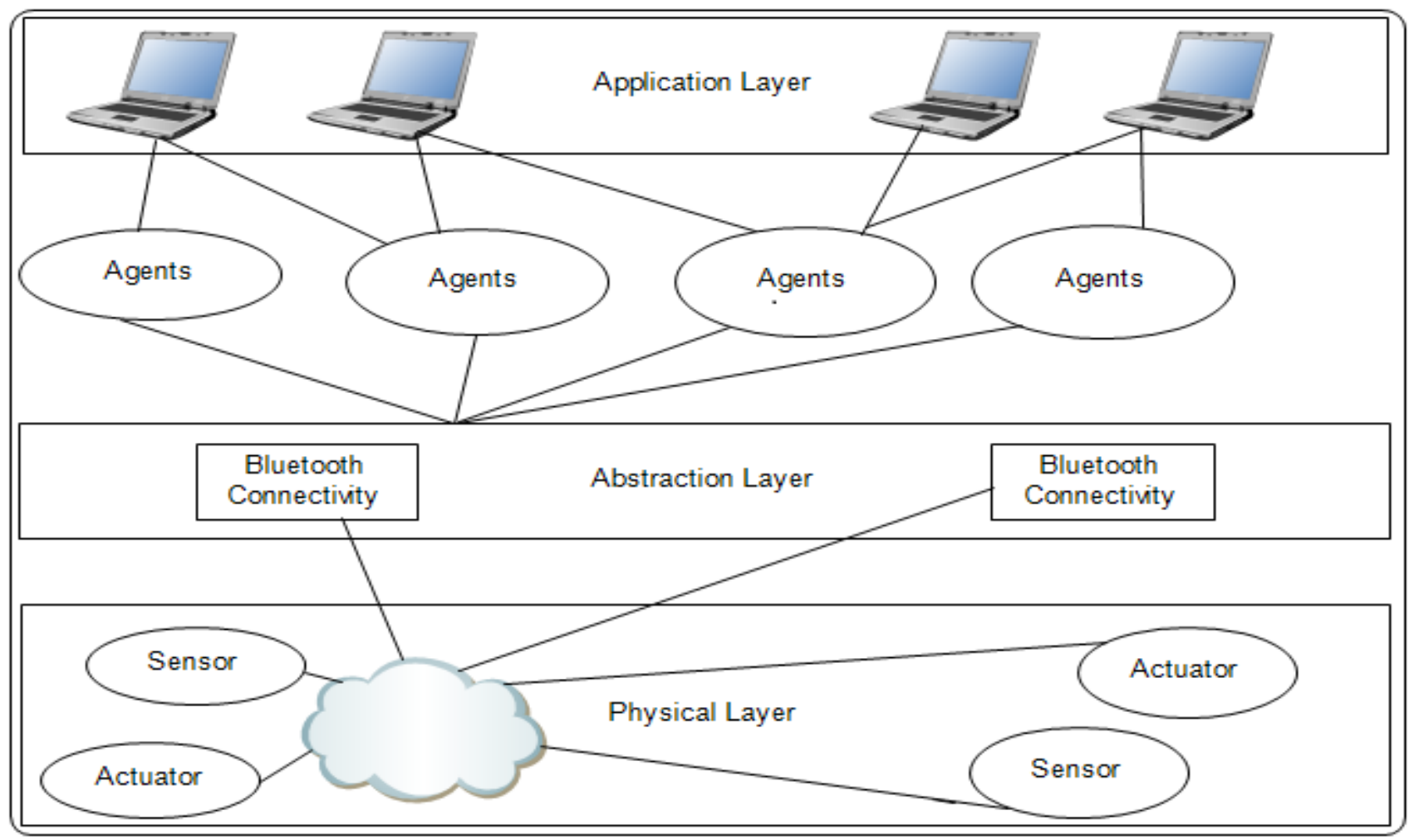

Fig1.ProposedArchitecture

Architectural Details are as follows:-

\subsubsection{Physical Layer}

This layer provides direct monitoring of sensors, actuators and control of field devices.

\subsubsection{Abstraction Layer}

An abstraction layer collects the control or data messages from the Physical layer and forwards them to the Agent layer. The communication between the abstraction layer and the physical layer providers is through wireless protocols.

\subsubsection{Agent Layer}

This layer provides an information, networking architecture for a sensor network application. It formulates requirements, maps them into requests to other agents for further processing and provides users with answers from the application agents. The user can communicate with any agent to express its needs.

\subsubsection{Application Layer}

It contains applications that periodically generate and cancel requirements for remote sensors and actuators by reevaluating the membership.

\subsection{Case Study}

Smart Campus: In a campus environment, a number of users share a large amount of their information needs. These needs include information about schedules, locations of classrooms, lectures, assignments, lab equipments, presentations, seminars, sports events, student ads, etc. Much of this information is directly related to objects, places and people that are situated within the campus environment. The exhaustive installation of wireless computing facilities such as WLAN, WSN together with small handheld devices and technologies for detecting objects or locations make it possible to satisfy the information needs of users in a campus environment in new ways. By embedding physical hyperlinks into the campus and attaching information to physical objects, visible entry points into the information space are created, enabling a natural interaction between the physical and the virtual environment and thus providing ubiquitous access to it. By linking the virtual to the real world, new interaction patterns emerge on both sides. This system focus on the aspect of linking virtual and physical elements in a campus environment. Smart campus provides the following functions:

\subsubsection{Classroom Occupancy}

The electric appliance in the classrooms was being left on for a long period of time while the rooms unoccupied. Despite of having individuals turned the light off as they leave the classroom the problem persisted and the college experienced wasted energy and money. To stop this energy waste decided to use occupancy sensors.

Occupancy sensors control lights by turning lights on only when the classroom is occupied and turning lights off after a classroom is vacated. This decision to use sensors was based on the fact that occupancy sensors would perform to save energy without requiring any manual actions by occupants. Their function is automatic and this seems to be smart classroom.

\subsubsection{Area Access Monitoring and Controlling}

Restricted area which can be accessed by only respective staff members in the campus area. For example account center, CAP center etc.

\subsubsection{Attendance Monitoring System in class}

A proposal to save energy by automatically register the attendance of students as students enter the classroom through nearest field communication (NFC) Sensor. By the use of this sensor the work of manual attendance will be minimized. 


\section{SYSTEM IMPLEMENTATION}

\subsection{Hardware interface}

Fig. 2 shows the hardware unit that is a combination of sensors and actuators to perform the defined above functions of smart campus

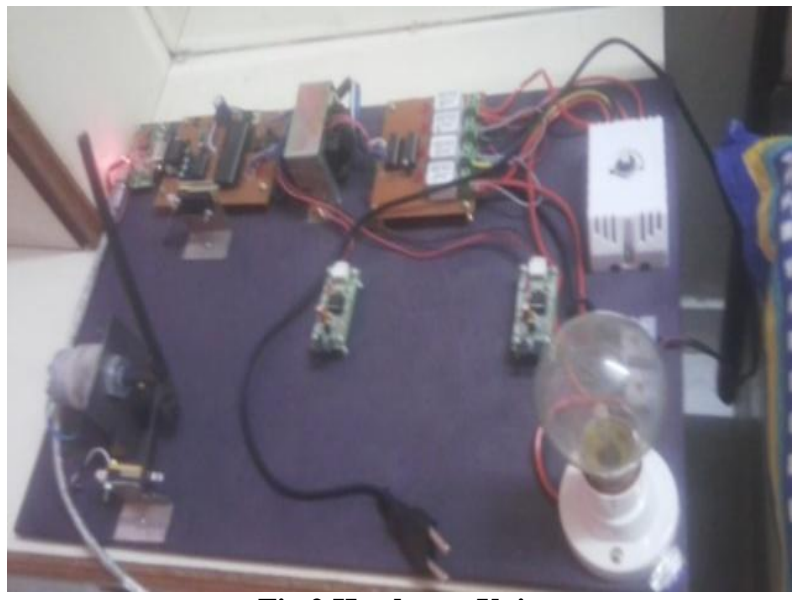

Fig.2 Hardware Unit

\subsection{Hardware Details}

Hardware interface details are as follows:

7.2.1 8-bit Microcontroller with $8 \mathrm{~K}$ bytes InSystem programmable Flash AT89S52

The AT89S52 is a low-power, high-performance CMOS 8-bit microcontroller with $8 \mathrm{~K}$ bytes of in-system programmable Flash memory. It provides the following standard features:

i. $8 \mathrm{~K}$ bytes of Flash

ii. 256 bytes of RAM

iii. 32 I/O lines, Watchdog timer, two data pointers, three 16bit timer/counters

\subsubsection{8-Bit P Compatible A/D Converters with 8- Channel Multiplexer ADC0808/ADC0809}

The ADC0808, ADC0809 data acquisition component is a monolithic CMOS device with an 8-bit analog-to-digital converter, 8-channel multiplexer and microprocessor compatible control logic.

Below are the following features:

i. Easy interface to all microprocessors.

ii. Operates ratio metrically with 5 VDC.

iii. Adjusted voltage reference.

iv. No zero or full-scale adjust required.

v. 8-channel multiplexer with address logic.

vi. $0 \mathrm{~V}$ to $\mathrm{VCC}$ input range.

\subsubsection{Leone Relay-SC5-Ag}

Its specifications are as follows:

i. Relay type: SC5

ii. Contact Material- Ag Alloy

iii. Coil Voltage- 3 48VDC

iv. Coil power Consumption- $0.36 \mathrm{~W}$

\subsubsection{Obstacle Detecting Sensor}

It is used to detect obstacles in front of sensor in applications. Sensor keeps transmitting modulated infrared light and when any object comes near, it is detected by the sensor by monitoring the reflected light from the object. Its features are as:-

i. Power Supply: 5V DC Power Consumption: 50mA max.

ii. Detection range $10 \mathrm{~cm}$

iii. Operation range varies according to the color of the object, light color has more range.

iv. Detection Indicator LED

\subsubsection{Nearest Field Communication(NFC) Sensor}

NFC is a smart card reader used to detect the presence of students in case study of smart campus. This sensor is used for the function of attendance monitoring in the classroom and area access controlling.

ACR122T NFC Contact less Smart Card Reader :

i. CCID-compliant

ii. PC/SC-compliant

iii. $\mathrm{Read} / \mathrm{write}$ speed up to $424 \mathrm{kbps}$

iv. Built-in antenna for contactless tag access, with card reading distance of up to $50 \mathrm{~mm}$

v. Support ISO 14443 Type A and B, Mifare, FeliCa and all 4 types of NFC (ISO/IEC 18092) tags

vi. Support all 3 modes of the NFC: reader, card emulation and peer-to-peer modes

\section{RESULT}

The Smart Campus application takes care of the following functions such as classroom occupancy, attendance monitoring and area access controlling.

This application requests for sensor access which transfers data when connected to cyber layer through the network.

Snapshot in fig. 4 shows the function of classroom occupancy. As occupancy increases in classroom it automatically controls the speed of the fan. When in-sensor detects the number of occupants from 1 to 3 control speed is set to 1 ,for 3 to 6 control speed is set to 2 and for 7 to 9 control speed is set to 3 . When there are no ooccupants ,the application returns 0 which automatically switches off the fan. With the use of this function, the energy consumption is minimized.

The second function is an attendance monitoring system in the classroom means as students enter the classroom the attendance gets automatically registered through NFC (Nearest Field Communication) sensor. Students filled registration form at the time of admission .It automates the manual process of attendance. Authentic data is generated. This is shown in fig.5 .This function helps to improve response time.

The third function is an area access controlling and monitoring system means it is required to fill the registration form for staff as shown in fig.6, whoever the campus administrator want to give them the access to specific areas. After filling the registration form, system will give the access pin number that is unique for every staff member.Through a pin number only, staff can access the critical areas of campus. 


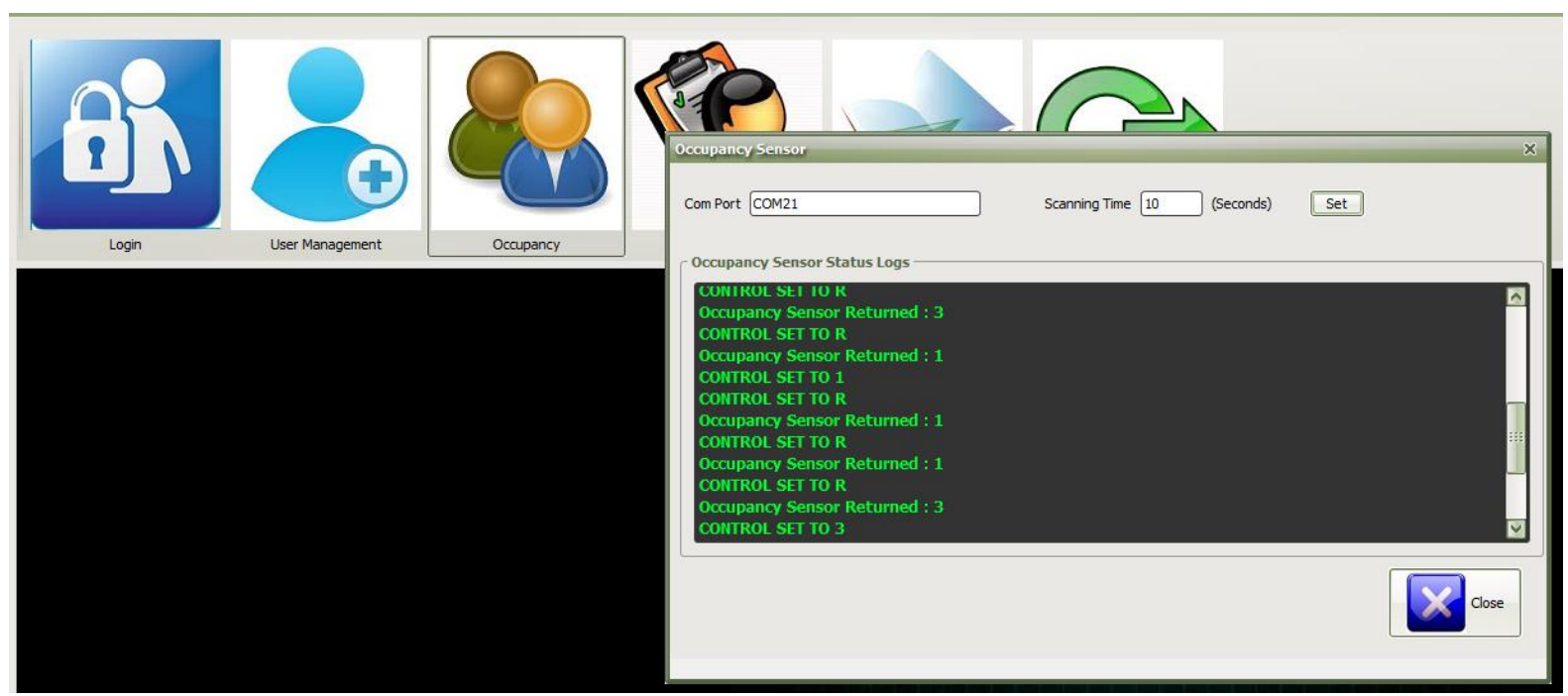

Fig.4 Control the speed of fan based on occupancy sensor returned

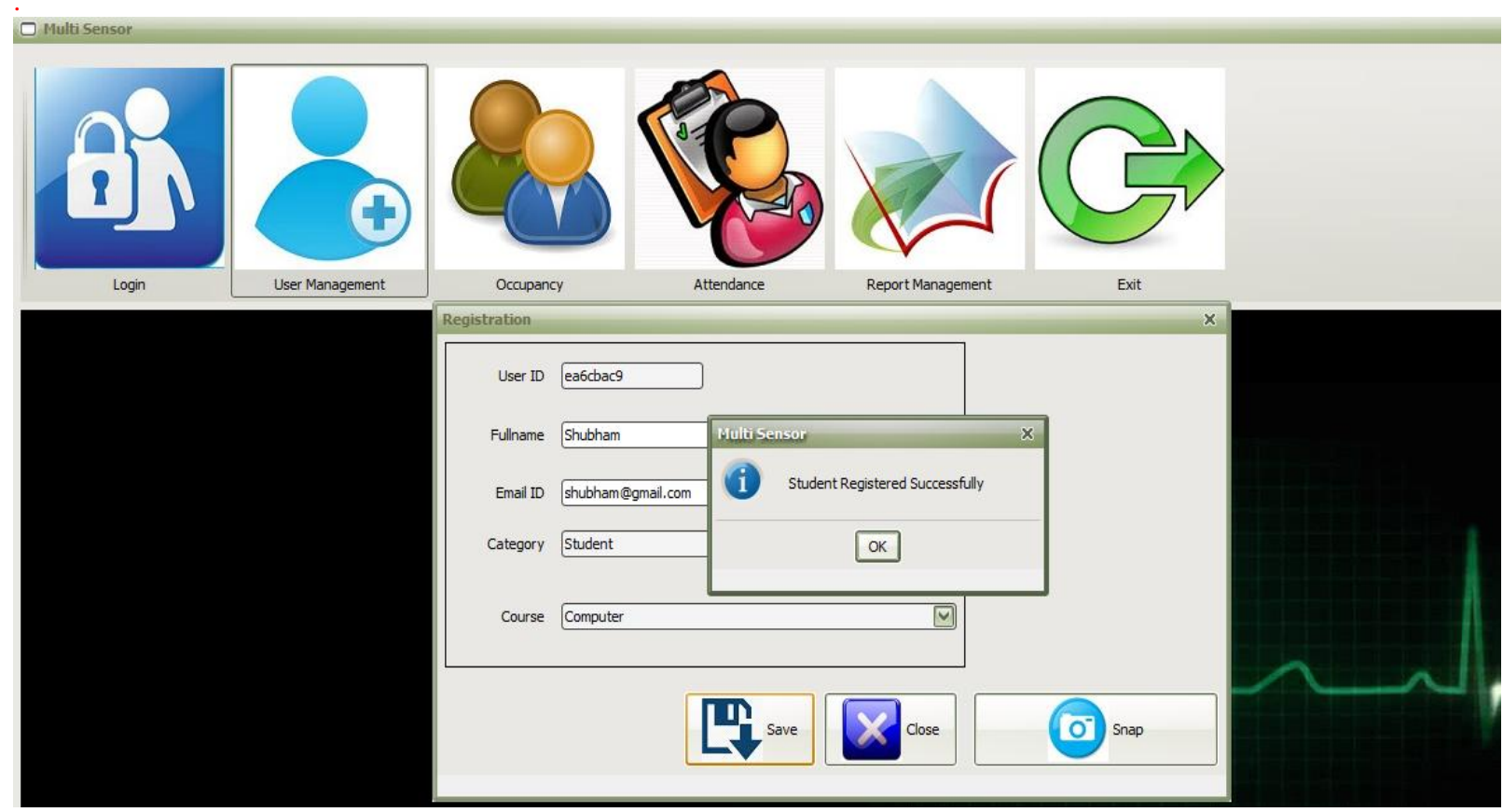

Fig.5 Registration form for students 


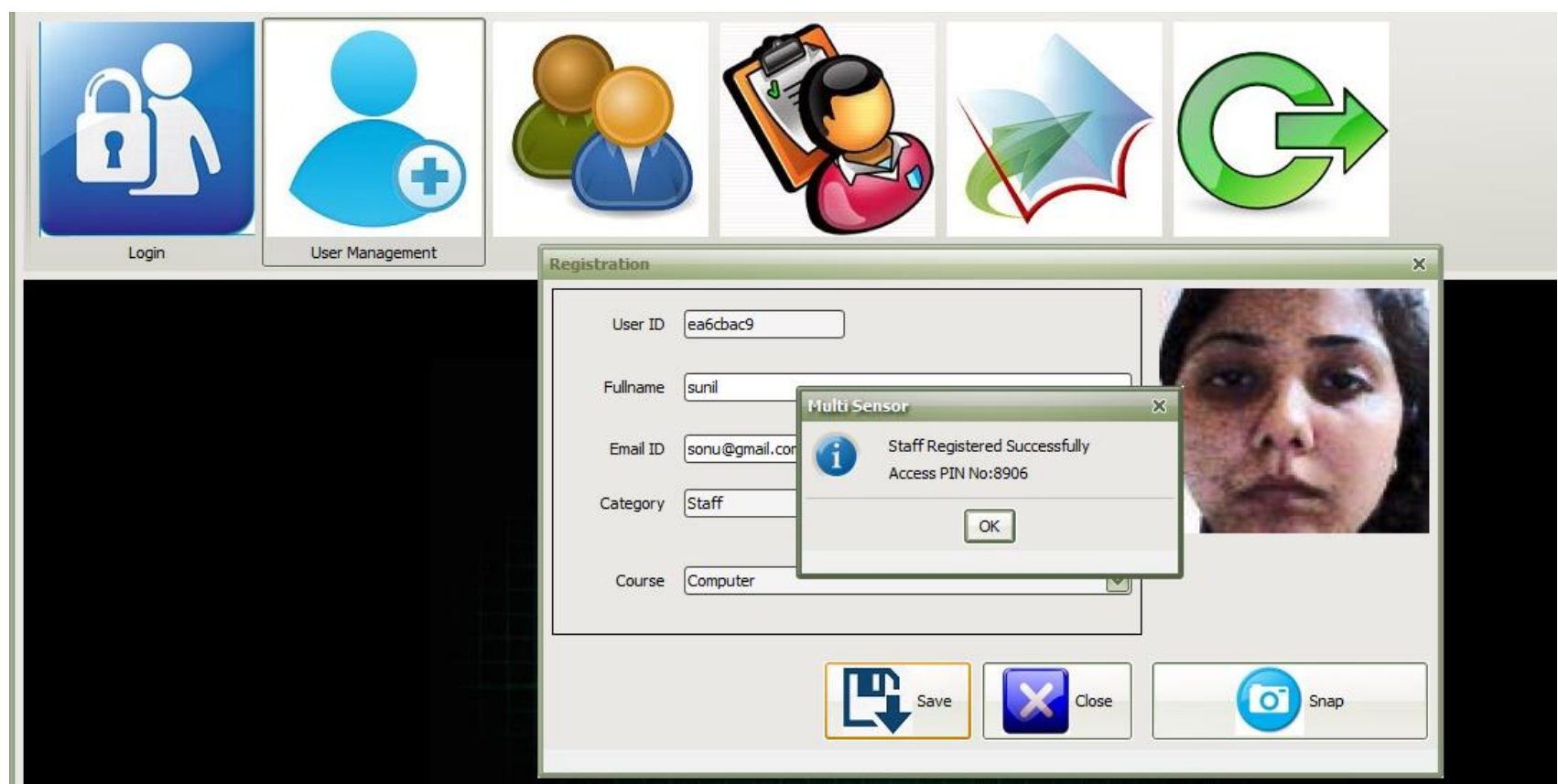

Fig.6 Staff Registration Form

\section{RESULT ANALYSIS}

Results show that the proposed in-network mechanisms can facilitate the efficient use of constrained resources and satisfy data requirements in the presence of dynamics and uncertainty. The goal of the programming system being developed as part of this research is to provide abstractions and mechanisms to seamlessly access and integrate remote and distributed sensor data into computational models and support scalable in-network data processing. The underlying approach is to virtualize the physical sensor to match the representation of the physical domain used by the models, and dynamically discover and access sensor data independently of any change to the sensor network itself. Below table1 shows that in earlier systems, there is a limitation of innetwork aggregation, which results in more energy consumption due to its centralized and distributed architecture. Now in the proposed system, it minimizes delay through which energy consumption is minimized using the concepts of an agent based approach for improving the lifetime of the network. It determines the value of the power consumption of sensors and actuators that are used in hardware unit.

Table 1 Energy Consumption between earlier system and proposed system

\begin{tabular}{|l|l|l|l|l|}
\hline $\begin{array}{l}\text { Sr. } \\
\text { No. }\end{array}$ & $\begin{array}{l}\text { No. } \\
\text { Sensors and } \\
\text { Actuators }\end{array}$ & $\begin{array}{l}\text { Delay } \\
\text { Energy } \\
\text { Consumpti } \\
\text { on } \\
\text { (Earlier } \\
\text { System) }\end{array}$ & $\begin{array}{l}\text { Energy } \\
\text { Consump } \\
\text { tion } \\
\text { Proposed } \\
\text { System) }\end{array}$ \\
\hline 1 & 7 & 300 & 361.4403 & $\begin{array}{l}361.439 \\
7\end{array}$ \\
\hline 2 & 14 & 300 & 722.8806 & $\begin{array}{l}722.879 \\
7\end{array}$ \\
\hline
\end{tabular}

Below the graph in fig.7 shows that in earlier systems, there is a limitation of in-network aggregation,which results in more energy consumption due to its centralized architecture. Now in the proposed system, it is shown that energy consumption is reduced to enhance the lifetime of the network using the concepts of an agent based approach.

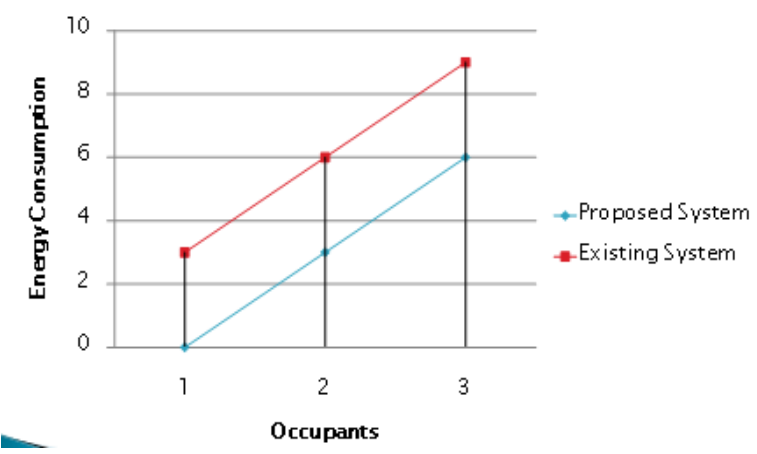

Fig.7 Energy efficient in proposed system

\section{CONCLUSION}

With the objective of maximizing the network lifetime, it considered the problem of in-network aggregation and local processing. An agent based approach, termed Improved group based programming abstraction for cyber physical system is proposed to solve the problem. It is managed and programming across pervasive computing networks, but with important capabilities for across system programming, mobility, automatic dynamic updates, fine grained access right control and conflict resolution mechanism, and support for actuators. Supports for both intra and inter network mobility and multiple application using the same sensor and actuator. It also facilitates in-network aggregation and local processing so that energy consumption and response time will be improved. It would validate on the application of smart campus, which considers following functions like classroom occupancy, attendance monitoring and area access controlling and monitoring.

\section{REFERENCES}

[1] Pascal A. Vicaire, Enamul Hoque, Zhiheng Xie, and John A. Stank Vic, "Bundle: A Group-Based 
programming Abstraction for Cyber-Physical Systems",IEEE Transactions on Industrial informatics, Volume No.8, ISSN: 1551-3203, pages 379-392, MAY 2012.

[2] Kamin Whitehouse, Cory Sharp, Eric Brewer, David Culler, "Hood:A Neighborhood Abstraction for Sensor Networks", 2nd International Conference on Mobile System Application Services, ISSN 1927-064, pages99$110,2004$.

[3] Luca Mottola and Gian Pietro Picco, "Logical Neighborhoods: A Programming Abstraction for Wireless Sensor Networks", Second IEEE International Conference, Volume 4026, ISSN 0302-9743, pages150168,2006

[4] Matt Welsh and Geoff Mainland, "Programming Sensor Networks Using Abstract Regions", 1st conference on Symposium on Networked Systems Design and Implementation, Volume 1 ,ISSN 1041-4347, pages 29-42, 2004

[5] Ryan Newton,Matt Welsh, "Region Streams: Functional Macroprogramming for Sensor Networks", ACM, Data Management for Sensor Networks, Volume 32, ISSN: 1556-4665, pages 78-87, 2004.

[6] Yang Ni, Ulrich Kremer, and Liviu Iftode, "Spatial Views: Space-Aware Programming for Networks of Embedded Systems", 16th International Workshop Languages Compilers Parallel Computing, Volume 2958, ISSN: 0302-9743, pages 258-272, 2003

[7] L. Luo, T. F. Abdelzaher, T. He, and J. A. Stankovic, "EnviroSuite: An Environmentally Immersive Programming Framework for Sensor Networks", ACM Transactions on Embedded Computing System, Volume 5, ISSN: 1539-9087,Pages 543-576, 2006.
[8] D. Jacobi, P. E. Guerrero, I. Petrov, and A. Buchmann, "Structuring sensor networks with scopes", 3rd IEEE European Conference on Smart Sensing and Context (EuroSSC), IEEE Communications Society, Oct. 2008.

[9] E. Cheong, J. Liebman, J. Liu, and F. Zhao, "Tiny gals: A programming model for event-driven embedded systems", ACM Symposium Application Computing, Volume18, pages 698-704, 2003.

[10] Fang-Jinx Wua, Yu-Fen Kaob, Yu-Chee Tseng, "From wireless sensor networks towards cyber physical systems", Journal of Pervasive and Mobile Computing, Volume 7, pages 397-413, August 2011.

[11] P. A. Vicaire, Z. Xie, E. Hoque, and J. A. Stankovic, "Physicalnet: A generic framework for managing and programming across pervasive computing networks", in Proc. 16th IEEE Real-Time Embedded Technology Application System, pages 269-278, 2010.

[12] Edward Ashford Lee, Sanjit Arun kumar Seshia, "An Introduction to Embedded System- A Cyber Physical System Approach", UC Berkley, First Edition, http://LeeSeshia.org.

[13] Teodora Sanislav, Liviu Miclea, "An Agent-oriented Approach for Cyber- Physical System with Dependability Features", IEEE International Conference on Automation Quality and Testing Robotics (AQTR), pages 356-361, 2012.

[14] Teodora Sanislav, Liviu Miclea, "Cyber-Physical Systems - Concept, Challenges and Research Areas", CEAI, Vol.14, No.2, pp. 28-33, 2012.

[15] Pooja Sobhrajan, Swati Nikam "Comparative Study of Abstraction in Cyber Physical System", (IJCSIT) International Journal of Computer Science and Information Technologies, Vol. 5 (1), ISSN 0975-9646, pp.466-469,2014. 